\title{
Testosterone reduced methylprednisolone-induced muscle atrophy in spinal cord-injured rats
}

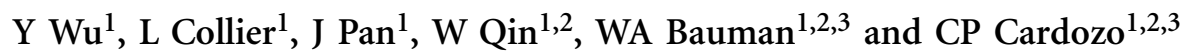

Study design: Male rats with complete transections of the spinal cord were administered vehicle or methylprednisolone (MP) for $24 \mathrm{~h}$, with or without infusion, for 7 days, of testosterone at either a replacement or low pharmacological doses. Muscles were collected at 7 days after SCl.

Objective: The objective of this study is to determine, in a rat model of complete spinal cord transection, whether testosterone reduces muscle atrophy or upregulates muscle atrophy-linked genes, induced by $24 \mathrm{~h}$ of MP administration at doses comparable to those prescribed in man during the period immediately following acute spinal cord injury ( $\mathrm{SCl}$ ) in an attempt to preserve neurological function. Results: MP significantly reduced the mass of triceps, soleus and plantaris, and significantly increased expression of genes that promote atrophy. Testosterone significantly reduced muscle atrophy induced by MP, but did not prevent it; there was no difference between low- or high-dose testosterone in reducing MP-induced muscle loss. High-dose testosterone reduced expression of muscle atrophy genes more than did low dose. Testosterone-induced declines in mRNA levels for these atrophy-associated genes did not correlate well with protection against MP-induced muscle atrophy.

Conclusions: MP induces marked and lasting changes in the biology of muscle that persisted for at least 7 days, or 6 days after MP has been eliminated from the body. Testosterone partially protected against muscle atrophy and gene expression changes caused by 1 day of MP.

Spinal Cord (2012) 50, 57-62; doi:10.1038/sc.2011.91; published online 20 September 2011

Keywords: Spinal cord injury; methylprednisolone; muscle atrophy; atrogenes; testosterone

\section{INTRODUCTION}

The glucocorticoid methylprednisolone (MP) is often administered at high doses, beginning immediately after spinal cord injury (SCI), to improve neurological outcomes. In the NASCIS II study, MP administered for $24 \mathrm{~h}$ improved motor, pinprick and touch at 6 months after injury, ${ }^{1}$ without significant effects on morbidity or mortality. In the NASCIS III study, administration of MP for $48 \mathrm{~h}$ was found to be superior to $24 \mathrm{~h}$ when treatment was initiated at later times $(3-8 \mathrm{~h}$ after injury), ${ }^{2}$ but 48 -h administration of MP increased the risk of sepsis and pneumonia compared with $24 \mathrm{~h}$. In a separate study, MP increased the risk of infection and hyperglycemia during the initial intensive care unit stay. ${ }^{3}$ Concerns have been raised regarding the potential for developing the well-recognized adverse effects of glucocorticoids on skeletal muscle, ${ }^{4}$ which include muscle atrophy ${ }^{5}$ and glucocorticoid myopathy. ${ }^{4}$ Although specific adverse effects of MPinduced muscle atrophy have not been studied in patients with SCI, one can envision reduced ability to transfer, relieve pressure and operate a wheel chair. Support for these concerns comes from findings that in SCI rats, this regimen of MP induced a more than 50\% decrease in triceps muscle weights at 7 days after injury, even though this muscle remained neurologically intact. ${ }^{6}$ In a case series, five of six patients, who were administered MP, had histological and electromyelographic evidence of myopathy, whereas none of those not administered this agent demonstrated these abnormalities. ${ }^{4}$ Such findings suggest that administration of very high doses of MP damages skeletal muscle.
Glucocorticoids increase muscle protein catabolism and reduce muscle protein synthesis and endurance. ${ }^{7}$ The degree of atrophy of skeletal muscle induced by glucocorticoids varies with muscle fiber type. In studies of the diaphragm, short-term administration of glucocorticoids did not alter the distribution of muscle fiber types. ${ }^{8}$ Significant atrophy of type II (fast-twitch) fibers was observed, ${ }^{7,8}$ whereas type I (slow-twitch) fibers were either unaffected $^{8}$ or less severely atrophied. ${ }^{7}$

Many of the adverse effects of glucocorticoids on skeletal muscle are attenuated by testosterone. ${ }^{5,9}$ Testosterone attenuates glucocorticoidinduced changes in gene expression in cell culture and animal models. ${ }^{5,9}$ Thus, testosterone prevents effects of glucocorticoids to upregulate a large number of genes in skeletal muscle that include several with clear roles in promoting muscle atrophy: muscle atrophy F-box (MAFbx), muscle ring finger 1 (MuRF1), forkhead box 01 (FOXO1) and regulated in development and DNA damage responses 1 (REDD1). ${ }^{10-12}$ MAFbx and MuRF1 are muscle-restricted E3 ubiquitin ligases that accelerate the rate of denervation atrophy. ${ }^{12}$ MuRF1 has been shown to be responsible for the selective decreases in myosin heavy chain levels that occur during glucocorticoid-induced atrophy. ${ }^{13}$ REDD1 is an inhibitor of protein synthesis. ${ }^{11}$ The transcription factor FOXO1 is both upregulated and activated by glucocorticoids, and upregulates MAFbx, MuRF1 and REDD1..$^{10,14-15}$

The protective actions of endogenous testosterone are likely to be diminished after SCI in men, particularly during the first few weeks or

${ }^{1}$ Department of Veterans Affairs, Center of Excellence for the Medical Consequences of Spinal Cord Injury, James J Peters VA Medical Center, Bronx, NY, USA; ${ }^{2}$ Department of Medicine, Mount Sinai School of Medicine, New York, NY, USA and ${ }^{3}$ Department of Rehabilitation Medicine, Mount Sinai School of Medicine, New York, NY, USA

Correspondence: Dr CP Cardozo, Department of Veterans Affairs, Center of Excellence for the Medical Consequences of Spinal Cord Injury, Room 7A-13, James J Peters VA Medical Center, 130 West Kingsbridge Road, Bronx, NY 10468, USA.

E-mail: Chris.Cardozo@mssm.edu

Received 13 May 2011; revised 25 July 2011; accepted 26 July 2011; published online 20 September 2011 
months after acute injury, because circulating levels of testosterone are often markedly reduced. ${ }^{16,17}$ Administration of glucocorticoid medications themselves have been associated with reduced circulating levels of testosterone, and these agents may produce a state of relative or absolute hypogonadism, ${ }^{18,19}$ which may be speculated to worsen any state of hypogonadism already present as a result of the stress of trauma or other medical conditions associated with SCI.

In the present study, we characterize the effects of testosterone on the adverse actions of MP on skeletal muscle mass and gene expression in a rat model of SCI. Our study included an examination of changes in expression of MAFbx, MuRF1, REDD1 and FOXO1, as well as evaluation of effects of MP alone, or together with testosterone weights of muscles with different fiber type compositions. As approximately $80 \%$ of persons with SCI are males, these studies were performed using male rats.

\section{MATERIALS AND METHODS}

\section{Animal studies}

All applicable institutional and governmental regulations concerning the ethical use of animals were followed during the course of this research. Animal studies were approved by the Institutional Animal Care and Use Committee at the James J Peters VA Medical Center. Specific pathogen-free male Wistar rats obtained from Taconic Farms (Hudson, NY, USA) and weighing 250-300 g were kept in a temperature and humidity-controlled room with a 12:12 h light:dark cycle. Anesthesia was induced and maintained by inhalation of isofluorane. The spinal cord was completely transected at the interspace between the 9th and 10th thoracic vertebra after exposure of the spinal cord by a laminectomy. A surgical gel sponge was placed between the ends of the transected spinal cord to stop bleeding. Wounds were closed in two layers using suture. Sham-SCI animal received an identical surgery, except that the spinal cord was not cut.

Animals were administered an intravenous injection of MP $\left(30 \mathrm{mg} \mathrm{kg}^{-1}\right)$ or vehicle (propylene glycol) by tail vein. An Alzet model 2001 pump (Durect, Cupertino, CA, USA) was implanted in a subcutaneous pocket over the back; this pump provided a $24-\mathrm{h}$ infusion of MP at $5.4 \mathrm{mg}^{-1} \mathrm{~kg} \mathrm{~h}^{-1}$, or vehicle. This dosing corresponds to that prescribed by the Bracken protocol to improve neurological outcomes after $\mathrm{SCI}^{20}$ on a $\mathrm{mg} \mathrm{kg}^{-1}$ basis. A second Alzet pump, model 2002, was also implanted, and administered either freshly prepared testosterone (2.8 or $7.5 \mathrm{mg} \mathrm{kg}^{-1}$ per day) or vehicle (propylene glycol). The lower testosterone dose corresponds to a replacement dose to achieve high physiological blood concentrations, ${ }^{21}$ whereas the higher dose of testosterone results in supraphysiological concentrations.

Urine was manually expressed at least three times daily. Animals were administered Baytril subcutaneously during the entire postoperative period. Muscles were removed, weighed, frozen in liquid nitrogen, and then stored at $-80^{\circ} \mathrm{C}$. Numbers per group were: Sham-SCI, 18 ; SCI-Vehicle, 12; SCI-MP, 12; SCI-MP+Ts, 7.5 (high dose), 14; and SCI-MP+Ts 2.8 (low dose), 7. Data for some animals from the Sham-SCI, SCI-Vehicle and SCI-MP groups have been included in a prior report ${ }^{6}(10,6$ and 9 animals for Sham-SCI, SCI-Vehicle and SCI-MP, respectively). The design of the two studies is shown in Figure 1.

\section{Extraction of total RNA and quantitative real-time PCR}

Extraction of total RNA from skeletal muscle and quantitative real-time PCR were performed, as previously described., ${ }^{9,22}$ Concentrations of RNA were determined by absorbance at $260 \mathrm{~nm}$, using a Nanodrop 1000 spectrophotometer (NanoDrop Products, Wilmingon, DE, USA). Real-time PCR determinations were performed using Assays on Demand available from Applied Biosystems (Carlsbad, CA, USA). Changes in expression levels of mRNA transcripts were expressed as fold change, as compared with the Sham-SCI group treated with vehicle using the $2^{-\Delta \Delta \mathrm{Ct}}$ method. The internal control for these analyses was $18 \mathrm{~S}$ RNA.

\section{Statistics}

Data are expressed as mean values \pm s.e.m. Values more than two s.d. from the mean were excluded during statistical analysis. The significance of differences among means was determined by a one-way analysis of variance, with a Tukey's
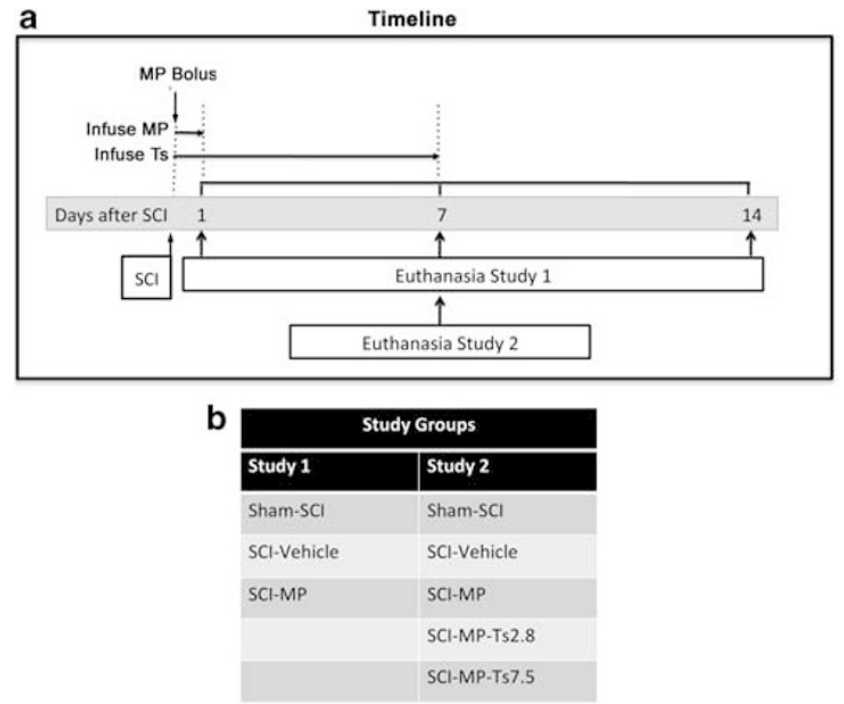

Figure 1 The design of Study 1 , which was previously reported, ${ }^{6}$ and of Study 2 are shown. Animals and tissues from the 7-day-time point in Study 1 were included for the analysis in Study 2. (a) The time line for SCl surgery, administration of a bolus of MP intravenously, and subsequent infusion of MP alone or together with testosterone is depicted. The times at which animals were euthanized for sample collection are also indicated for each study. (b) The groups included in studies 1 and 2 are listed.

test post hoc to establish the significance of differences between specific pairs of means. Statistical calculations were performed with Prism 4.0c (Graphpad Software, La Jolla, CA, USA).

\section{RESULTS}

\section{Body and muscle weights}

Consistent with a previous report, ${ }^{6} \mathrm{MP}$ significantly reduced body weight $(P<0.001)$ and the wet weights of triceps, plantaris, soleus and gastrocnemius, compared with weights for SCI-Vehicle animals $(P<0.01$, Figure 2). Triceps was chosen for these studies as a major weight-bearing muscle of the forelimb, and gastrocnemius was selected, because like triceps, it is an extensor and has a mixed fiber type. Soleus and plantaris were included, because they represent examples of primarily slow- and fast-twitch fibers, respectively.

Testosterone at both the low and high dose significantly attenuated by approximately $40 \%$ the loss of body weight of MP-treated animals $(P<0.05$, Figure 2a). There was no significant difference between the effects of low- and high-dose testosterone on body weight (Figure 2a). Although body weights for animals, administered either dose of testosterone, remained lower than those for SCI-Vehicle animals, this difference was not significant (Figure 2a).

Weights of triceps muscles, which remain under voluntary control after mid-thoracic SCI, were significantly reduced by approximately $50 \%$ by MP compared with SCI-Vehicle $(P<0.001$, Figure $2 b)$. Weights of the gastrocnemius, soleus and plantaris were also significantly reduced by MP by an additional 10 to $20 \%$, compared with muscle loss for SCI-Vehicle $(P<0.01$, Figures $2 \mathrm{~b}-\mathrm{e})$.

Both low- and high-dose testosterone significantly reduced triceps muscle atrophy caused by MP, by approximately two thirds $(P<0.001$, Figure 2b). No difference in triceps weights was observed when comparing low- and high-dose testosterone (Figure 2b). Weights of triceps from testosterone-treated SCI-MP animals remained significantly lower than weights for SCI-Vehicle animals, by approximately 


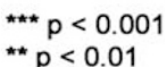

** $p<0.01$

a

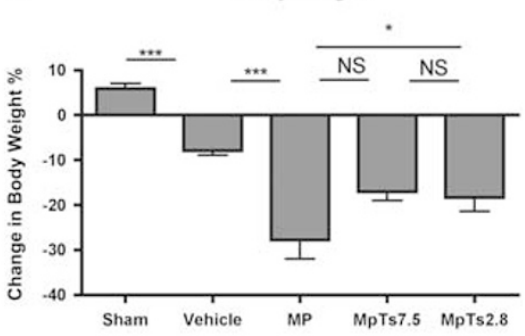

b

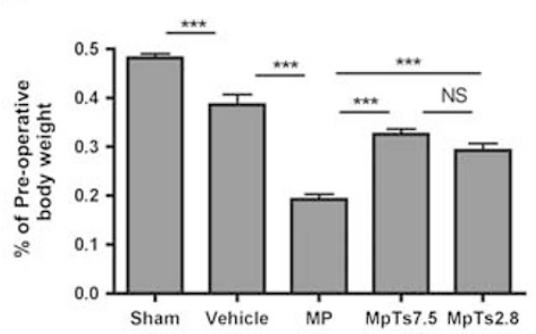

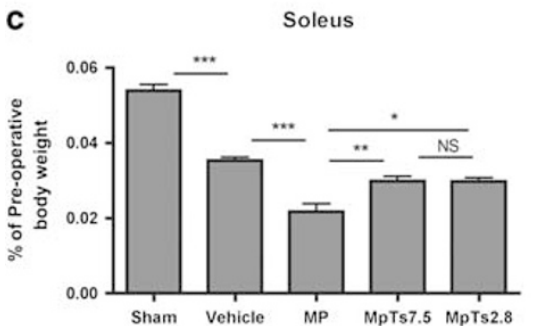
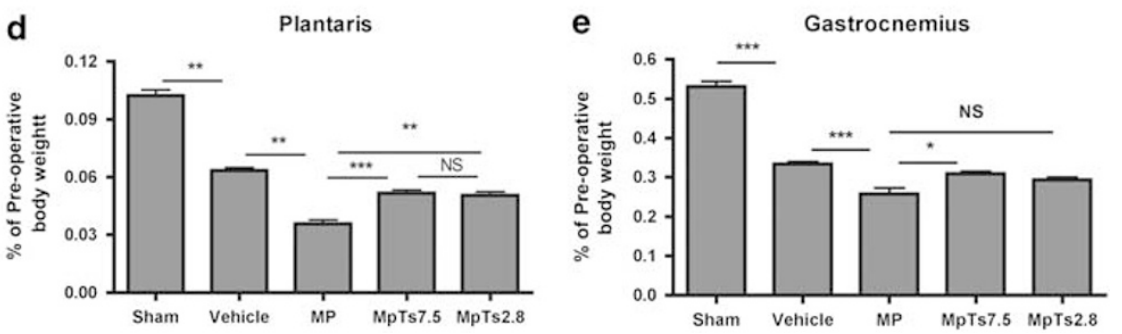

Figure 2 Effects of testosterone on MP-induced changes in body and muscle weights. (a) Change in body weight at 7 days expressed as a percentage of body weight measured before anesthesia. (b-e) Weights for skeletal muscle normalized to body weight before anesthesia. Note the legend to symbols used in the figure to the left of (a). Data are mean values \pm s.e.m. The numbers of animals per group were: SCI-Vehicle, 12; SCI-MP, 12; SCI-MP+Ts 7.5, 14; SCI-MP and Ts 2.8, 7.

a

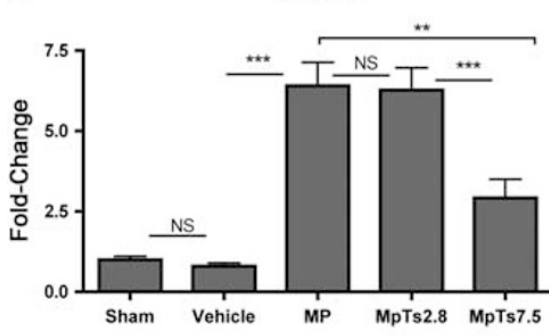

C

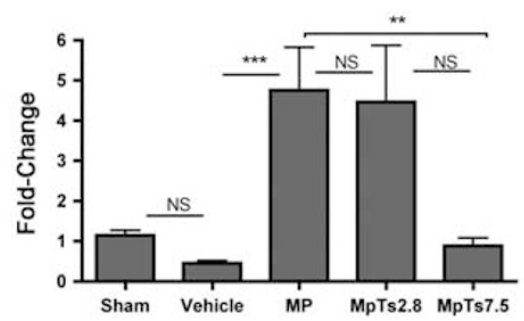

b

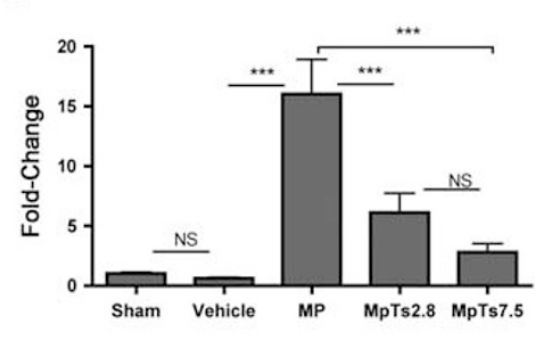

d

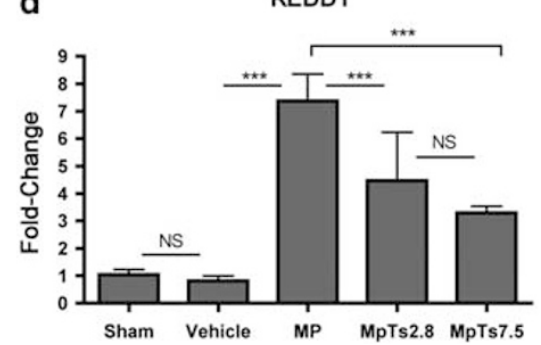

Figure 3 Levels of atrophy-related mRNAs in triceps muscle. (a-d). Levels of mRNA for MAFbx, MuRF1, FOXO1 and REDD1 are shown as fold-change relative to Sham-SCl as indicated above each panel, and are normalized to levels present in the Sham-SCl group. Data are mean values \pm s.e.m., and $\mathrm{N}$ is as in the legend to Figure 1. NS, non-significant difference; ${ }^{* *} P<0.01 ;{ }^{* *} P<0.001$.

16 and $24 \%$ for high- and low-dose testosterone, respectively. Both low- and high-dose testosterone significantly increased muscle weights for soleus $(P<0.01)$, plantaris $(P<0.001)$ and gastrocnemius $(P<0.05$; Figures $2 c-e)$. There was no significant difference in weights of these muscles from animals administered low or high doses of testosterone (Figures 2c-e). Weights of plantaris and gastrocnemius muscles from testosterone-treated animals administered high-dose testosterone remained significantly below those for SCI-Vehicle animals by $18 \%$ and $7 \%$, respectively $(P<0.05$; Figures $2 \mathrm{~d}$ and $\mathrm{e})$.
Weights of plantaris in the low-dose testosterone group were also reduced $(P<0.01)$ compared to the SCI-Vehicle group (Figures $2 \mathrm{~d}$ and e).

\section{Muscle gene expression}

At 7 days, expression of FOXO1, MAFbx, MuRF1 and REDD1 was not significantly altered in triceps muscle from the SCI-Vehicle group as compared with Sham-SCI (Figures 3a-d). A significant increase of 5- to 15-fold in expression of each of these genes was observed in triceps 

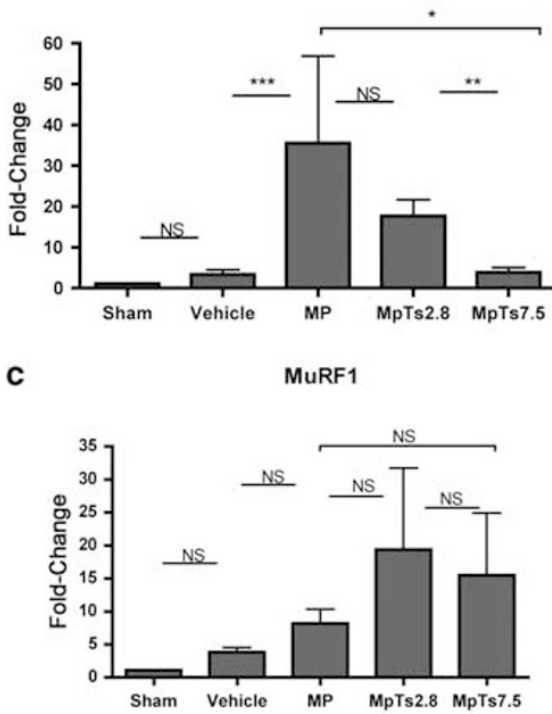

b

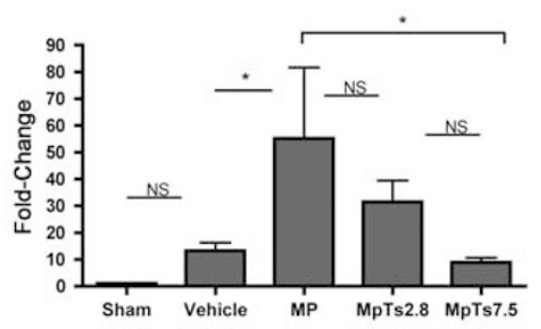

d

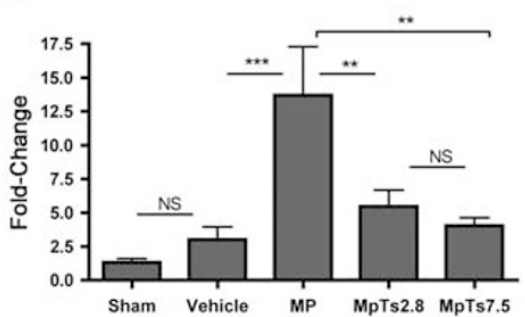

Figure 4 Levels of atrophy-related mRNAs in plantaris muscle. (a-d). Levels of mRNA for MAFbx, MuRF1, FOXO1 and REDD1 are shown as fold-change relative to Sham-SCl as indicated above each panel, and are normalized to levels present in the Sham-SCl group. Data are mean values $\pm \mathrm{s}$.e.m., and $\mathrm{N}$ is as in the legend to Figure 1 . NS, non-significant difference; ${ }^{*} P<0.05 ;{ }^{*} P<0.01 ; * * P<0.001$

from the animals in the SCI-MP group $(P<0.001$, Figures $3 \mathrm{a}-\mathrm{d})$. The higher dose of testosterone significantly reduced expression of each of these transcripts in triceps from SCI-MP animals by $50 \%$ for FOXO1 to more than $90 \%$ for MuRF1 $(P<0.01)$, although levels of these mRNAs remained greater than those for SCI-Vehicle for all transcripts, except MuRF1 (Figures 3a-d). Low-dose testosterone significantly reduced mRNA levels for MAFbx and REDD1 $(P<0.001)$, but not for FOXO1 or MuRF1 (Figures 3a-d).

We next examined changes in gene expression in soleus and plantaris muscles to gain insight into effects of testosterone on paralyzed muscle. Soleus and plantaris are composed primarily of slow- or fast-twitch fiber types, respectively, and thus permit one to test for fiber type-specific effects. Expression of FOXO1, MAFbx, MuRF1 and REDD1 tended to be increased in plantaris from the SCI-Vehicle group, but this change was not significant. In the SCI-MP groups, significant increases in expression were observed for FOXO1, MAFbx and REDD1 $(P<0.05$, Figures $4 \mathrm{a}-\mathrm{d})$; increases ranged from 13-fold for REDD1 to more than 50-fold for MAFbx. High-dose testosterone significantly $(P<0.05)$ reduced mRNA levels for FOXO1, MAFbx and REDD1, to levels that were similar to those in the SCIVehicle group (Figures $4 \mathrm{a}-\mathrm{d}$ ). Low-dose testosterone significantly reduced expression of REDD1 only $(P<0.01$, Figure $4 \mathrm{~d})$; there was no significant difference between mRNA levels for REDD1 between the low- and high-dose testosterone groups. By contrast, in soleus, no significant changes in mRNA levels were observed for SCI-Vehicle, SCI-MP or SCI-MP/Ts 7.5 groups (Figure 5). The paradoxical increase in FOXO1 and MAFbx mRNA levels observed in the low-dose testosterone group was assumed to be a type II error.

\section{DISCUSSION}

\section{Nature of muscle loss due to MP}

The genes studied were selected, because there is strong evidence that each stimulates specific biochemical processes responsible for atrophy by increasing catabolism of existing proteins and inhibiting the synthesis of new ones. MAFbx and MuRF1 are muscle-restricted ubiquitin ligases known to target a growing list of proteins for destruction. ${ }^{23}$ Loss of either ligase reduces denervation atrophy in transgenic mice, and loss of MuRF1 prevents loss of myosin heavy chain during glucocorticoid-induced atrophy. ${ }^{12,13}$ Thus, expression of MAFbx and MuRF1 is necessary for atrophy to proceed at its normal rate. However, levels of mRNAs for these ligases correlates poorly with atrophy rates, ${ }^{24}$ suggesting that increases in their expression are not sufficient for atrophy. REDD1 is an inhibitor of mammalian target of rapamycin $\mathrm{C} 1$, a master regulator of cell size and protein synthesis. REDD1 is upregulated in skeletal muscle by glucocorticoids, ${ }^{9,11}$ suggesting one mechanism by which glucocorticoids reduce protein synthesis. Expression of REDD1, MAFbx and MuRF1 is upregulated by FOXO1, ${ }^{10,14,15}$ a transcription factor from the forkhead family. Transcriptional activity of FOXO1 in stimulated by glucocorticoids. ${ }^{10}$

Consistent with our prior report, at 7 days, MP induced marked atrophy of muscle above the level of the injury, and further increased the atrophy of the paralyzed muscles below the lesion. In addition, in the current study, we found that at 7 days, MP induced significant elevations in expression of four genes linked to muscle atrophy in triceps and of three of these genes in plantaris. These findings extend those of our prior study in which we observed significant elevation of FOXO1 at 7 days in triceps and trends for the elevation of MAFbx and REDD1 at this time point. Of note, group sizes were smaller in our previous study, which may explain, in part, why these trends for MAFbx and REDD1 did not reach significance. The persistent increased expression of FOXO1, MAFbx, MuRF1 and REDD1 in the present study is unlikely to be due to the presence of MP in the circulation, or tissues after cessation of infusion because of the short half-life of the drug. ${ }^{25}$ Thus, these findings suggest that MP induces lasting changes in the biology of skeletal muscle that persist for at least 6 days after cessation of drug administration, and result in sustained alterations in gene expression for factors that drive atrophy programs (FOXO1), promote catabolism of muscle proteins (MuRF1 and MAFbx), and inhibit muscle protein synthesis (REDD1). ${ }^{9-12}$

In plantaris, MP increased expression of MAFbx, FOXO1 and REDD1, whereas in soleus, no significant change in expression of these genes was observed. One likely interpretation of these findings is 


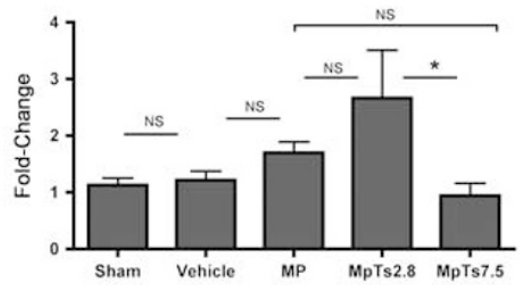

C

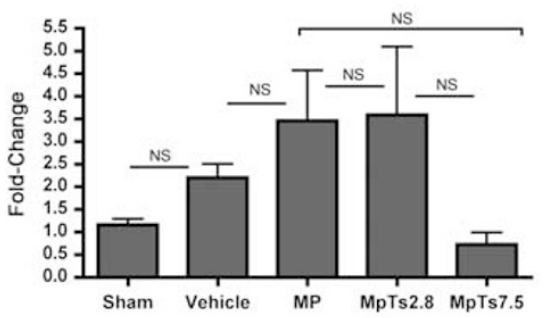

b

MAFbx

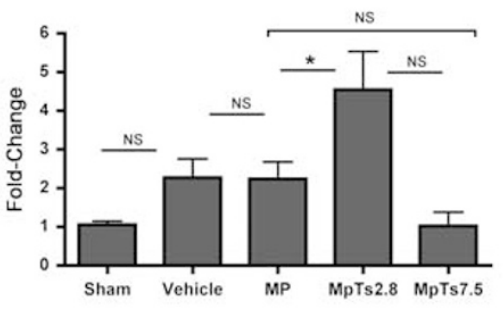

d

REDD1

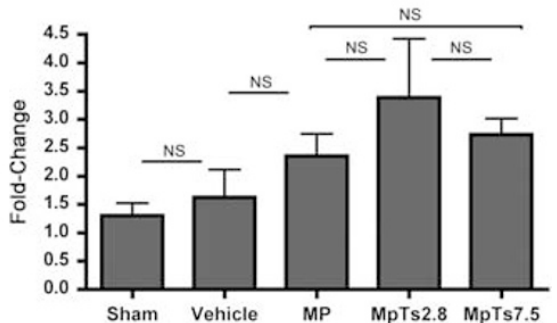

Figure 5 Levels of atrophy-related mRNAs in soleus muscle. (a-d) Levels of mRNA for MAFbx, MuRF1, FOXO1 and REDD1 are shown as fold-change relative to Sham-SCl as indicated above each panel, and are normalized to levels present in the Sham-SCl group. Data are mean values \pm s.e.m., and $\mathrm{N}$ is as in the legend to Figure 1 . NS, non-significant difference; ${ }^{*} P<0.05$.

that the effects of 1 day of high-dose MP on paralyzed and nonparalyzed muscle depends on fiber type. This conclusion is in agreement with reports addressing the effects of 2.5 or 10 weeks of glucocorticoid administration on diaphragm fiber cross-sectional areas, in which type II fibers were most greatly affected. ${ }^{7}$ Several other reports have found that tonic oxidative muscles are more resistant to glucocorticoid-induced atrophy than fast-twitch-rich muscles (for review, see Almon and Dubois ${ }^{26}$ ).

\section{Effects of Ts on MP-induced muscle loss}

At the time of greatest adverse effects of MP on muscle weights, testosterone reduced adverse effects of MP on body weight, muscle weights above and below the level of the SCI, and muscle gene expression. Testosterone prevented the atrophy of fast-twitch (plantaris), slow twitch (soleus) and mixed (gastrocnemius and triceps) fiber types induced by high-dose MP administration. Testosterone did not completely prevent the adverse effects of MP on muscle weights, even though the design of the studies favored any beneficial effects of this agent by extending the period of testosterone administration past the end of the period of MP administration. Although there was no difference in effects on muscle weights between the two testosterone doses when effects on gene expression were examined, the higher testosterone dose conferred more benefit. This effect was most apparent for triceps, but also evident for plantaris. Our study design and findings do not permit us to make any conclusions concerning the potential benefits to muscle function that might arise from the higher doses of testosterone.

Surprisingly, MP-induced expression of transcripts for MAFbx, MuRF1, FOXO1 and REDD1 was not tightly coupled to muscle atrophy at 7 days, as demonstrated by MP-induced atrophy of soleus despite minimal gene expression changes. The finding that low- and high-dose testosterone had similar activity in protecting against atrophy of triceps, soleus, plantaris and gastrocnemius, whereas the high dose was more effective in reducing MP-induced upregulation of
FOXO1, MAFbx, MuRF1 and REDD1 also indicates an uncoupling of elevation of the expression levels of these transcripts from that of muscle atrophy. A similar uncoupling has been found in animal models of muscle atrophy due to burns. ${ }^{24}$ Although increased levels do not correlate well with atrophy, the expression of MAFbx and MuRF1 do seem to be necessary for atrophy to occur. Denervation atrophy is slowed by disruption of either the MAFbx or MuRF1 genes, ${ }^{12}$ and glucocorticoid-induced loss of myosin heavy chain is prevented by a knockout for MuRF1. ${ }^{13}$

The protective effects of testosterone against most of the MPinduced atrophy of muscles above the level of SCI was consistent with those observed in studies in neurologically intact rats administered at a dose of dexamethasone that was approximately 30 -fold lower when expressed in glucocorticoid equivalents, 5,9 and which produced less severe atrophy. ${ }^{5}$ Our findings are also consistent with findings that another androgenic steroid, nandrolone, reversed glucocorticoid-induced diaphragm atrophy in rats. ${ }^{27}$ Effects of testosterone on expression levels of MAFbx, MuRF1, FOXO1 and REDD1 are also consistent with prior reports that testosterone attenuates dexamethasone-induced changes in expression of these genes. ${ }^{5,9}$

An interesting question is why testosterone was only partially effective in reducing muscle loss caused by MP after SCI. The likely answer is that at the doses of testosterone used in our study does not block all actions of glucocorticoids on muscle gene expression. Support for this view comes from a microarray analysis of the effects of testosterone on gene expression changes induced in rat skeletal muscle by dexamethasone, when both agents were administered for 7 days. This study found that only approximately 50\% of the gene expression changes induced by dexamethasone were blocked or attenuated by testosterone. ${ }^{9}$

Our analysis did not examine changes in two pathways also known to be altered by glucocorticoids, specifically that of insulin-like growth factor 1 and myostatin. Insulin-like growth factor 1 is an important growth factor for skeletal muscle, and levels of this growth factor fall in 
skeletal muscle after administration of glucocorticoids. ${ }^{28}$ Expression of myostatin is increased by glucocorticoids in some studies, ${ }^{29}$ and in transgenic mice, deletion of this gene prevents glucocorticoid-induced atrophy. ${ }^{30}$ Whether, and how much, each of these glucocorticoidinduced signals would be altered by concurrent administration of testosterone is not known. Several reports suggest that testosterone may have beneficial effects on these signaling pathways. The expression of insulin-like growth factor 1 was shown to be upregulated by testosterone in cell culture systems and skeletal muscle, ${ }^{31,32}$ and myostatin activation was reduced in a mouse model of aging-related sarcopenia. $^{33}$

\section{CONCLUSIONS}

Our findings highlight the marked and lasting impact of 1 day of MP administered at the time of acute SCI on skeletal muscle by demonstrating persistent elevation for at least 7 days in the expression of mRNA for several muscle atrophy genes. This work also demonstrates that testosterone, when administered at replacement doses, reduces muscle atrophy caused by MP. Higher doses of testosterone were also effective in reducing the MP-induced upregulation of the known muscle atrophy genes FOXO1, MAFbx and REDD1. When taken together with the findings that the trauma of acute SCI and that of glucocorticoid administration both serve to reduce circulating testosterone levels in men, ${ }^{17-19}$ our findings suggest the possibility that there may be a clinical benefit to the use of replacement doses of testosterone in spinal cord-injured men administered high-dose MP therapy at the time of injury. This possibility will be an important area for future study.

\section{CONFLICT OF INTEREST}

The authors declare no conflict of interest.

\section{ACKNOWLEDGEMENTS}

This research was supported by the Veterans Health Administration, Rehabilitation Research and Development Service (B4162C and B3347 K).

1 Bracken MB, Shepard MJ, Collins WF, Holford TR, Young W, Baskin DS et al. A randomized, controlled trial of methylprednisolone or naloxone in the treatment of acute spinal-cord injury. Results of the Second National Acute Spinal Cord Injury Study. N Engl J Med 1990; 322: 1405-1411.

2 Bracken MB, Shepard MJ, Holford TR, Leo-Summers L, Aldrich EF, Fazl M et al. Administration of methylprednisolone for 24 or 48 hours or tirilazad mesylate for 48 hours in the treatment of acute spinal cord injury. Results of the Third National Acute Spinal Cord Injury Randomized Controlled Trial. National Acute Spinal Cord Injury Study. JAMA 1997; 277: 1597-1604.

3 Suberviola B, Gonzalez-Castro A, Llorca J, Ortiz-Melon F, Minambres E. Early complications of high-dose methylprednisolone in acute spinal cord injury patients. Injury 2008; 39: 748-752.

4 Qian T, Guo X, Levi AD, Vanni S, Shebert RT, Sipski ML. High-dose methylprednisolone may cause myopathy in acute spinal cord injury patients. Spinal Cord 2005; 43: 199-203.

5 Zhao W, Pan J, Zhao Z, Wu Y, Bauman WA, Cardozo CP. Testosterone protects against dexamethasone-induced muscle atrophy, protein degradation and MAFbx upregulation. J Steroid Biochem Mol Biol 2008; 110: 125-129.

6 Wu Y, Hou J, Collier L, Pan J, Hou L, Qin W et al. The administration of high-dose methylprednisolone for $24 \mathrm{~h}$ reduced muscle size and increased atrophy-related gene expression in spinal cord-injured rats. Spinal Cord 2011; 49: 867-873.

7 Prezant DJ, Karwa ML, Richner B, Maggiore D, Gentry EI, Chung V et al. Short-term vs long-term dexamethasone treatment: effects on rat diaphragm structure and function. Lung 1998; 176: 267-280.

8 Lewis MI, Monn SA, Sieck GC. Effect of corticosteroids on diaphragm fatigue, SDH activity, and muscle fiber size. J Appl Physiol 1992; 72: 293-301.
9 Wu Y, Zhao W, Zhao J, Zhang Y, Qin W, Pan J et al. REDD1 is a major target of testosterone action in preventing dexamethasone-induced muscle loss. Endocrinology 2010; 151: 1050-1059.

10 Sandri M, Sandri C, Gilbert A, Skurk C, Calabria E, Picard A et al. Foxo transcription factors induce the atrophy-related ubiquitin ligase atrogin- 1 and cause skeletal muscle atrophy. Cell 2004; 117: 399-412.

11 Wang H, Kubica N, Ellisen LW, Jefferson LS, Kimball SR. Dexamethasone represses signaling through the mammalian target of rapamycin in muscle cells by enhancing expression of REDD1. J Biol Chem 2006; 281: 39128-39134.

12 Bodine SC, Latres E, Baumhueter S, Lai VK, Nunez L, Clarke BA et al. Identification of ubiquitin ligases required for skeletal muscle atrophy. Science 2001; 294: 1704-1708.

13 Clarke BA, Drujan D, Willis MS, Murphy LO, Corpina RA, Burova E et al. The E3 Ligase MURF1 degrades myosin heavy chain protein in dexamethasone-treated skeletal muscle. Cell Metab 2007; 6: 376-385.

14 Harvey KF, Mattila J, Sofer A, Bennett FC, Ramsey MR, Ellisen LW et al. FOXOregulated transcription restricts overgrowth of Tsc mutant organs. J Cell Biol 2008; 180: 691-696.

15 Waddell DS, Baehr LM, van den Brandt J, Johnsen SA, Reichardt HM, Furlow JD et al. The glucocorticoid receptor and foxol synergistically activate the skeletal muscle atrophy associated Murf1 gene. Am J Physiol Endocrinol Metab 2008; 295: E785-E797.

16 Tsitouras PD, Zhong YG, Spungen AM, Bauman WA. Serum testosterone and growth hormone/insulin-like growth factor-I in adults with spinal cord injury. Horm Metab Res 1995; 27: 287-292.

17 Schopp LH, Clark M, Mazurek MO, Hagglund KJ, Acuff ME, Sherman AK et al. Testosterone levels among men with spinal cord injury admitted to inpatient rehabilitation. Am J Phys Med Rehabil 2006; 85: 678-684; quiz 685-7.

18 Reid IR, Ibbertson HK, France JT, Pybus J. Plasma testosterone concentrations in asthmatic men treated with glucocorticoids. Br Med J (Clin Res Ed) 1985; 291: 574.

19 MacAdams MR, White RH, Chipps BE. Reduction of serum testosterone levels during chronic glucocorticoid therapy. Ann Intern Med 1986; 104: 648-651.

20 Bracken MB, Holford TR. Neurological and functional status 1 year after acute spinal cord injury: estimates of functional recovery in National Acute Spinal Cord Injury Study II from results modeled in National Acute Spinal Cord Injury Study III. J Neurosurg 2002; 96(3 Suppl): 259-266.

21 Borst SE, Lee JH, Conover CF. Inhibition of 5alpha-reductase blocks prostate effects of testosterone without blocking anabolic effects. Am J Physiol Endocrinol Metab 2005; 288: E222-E227.

22 Zhao J, Zhang Y, Zhao W, Wu Y, Pan J, Bauman WA et al. Effects of nandrolone on denervation atrophy depend upon time after nerve transection. Muscle Nerve 2008; 37: 42-49.

23 Glass D, Roubenoff R. Recent advances in the biology and therapy of muscle wasting. Ann NY Acad Sci 2010; 1211: 25-36.

24 Fareed MU, Evenson AR, Wei W, Menconi M, Poylin V, Petkova V et al. Treatment of rats with calpain inhibitors prevents sepsis-induced muscle proteolysis independent of atrogin-1/MAFbx and MuRF1 expression. Am J Physiol Regul Integr Comp Physiol 2006; 290: R1589-R1597.

25 Hazra A, Pyszczynski N, DuBois DC, Almon RR, Jusko WJ. Pharmacokinetics of methylprednisolone after intravenous and intramuscular administration in rats. Biopharm Drug Dispos 2007; 28: 263-273.

26 Almon RR, Dubois DC. Fiber-type discrimination in disuse and glucocorticoid-induced atrophy. Med Sci Sports Exerc 1990; 22: 304-311.

27 Van Balkom RH, Dekhuijzen PN, Folgering HT, Veerkamp JH, Van Moerkerk HT, Fransen JA et al. Anabolic steroids in part reverse glucocorticoid-induced alterations in rat diaphragm. J Appl Physiol 1998; 84: 1492-1499.

28 Inder WJ, Jang C, Obeyesekere VR, Alford FP. Dexamethasone administration inhibits skeletal muscle expression of the androgen receptor and IGF-1 - implications for steroid-induced myopathy. Clin Endocrinol (Oxf) 2009; 73: 126-132.

29 Ma K, Mallidis C, Bhasin S, Mahabadi V, Artaza J, Gonzalez-Cadavid N et al. Glucocorticoid-induced skeletal muscle atrophy is associated with upregulation of myostatin gene expression. Am J Physiol Endocrinol Metab 2003; 285: E363E371.

30 Gilson H, Schakman O, Combaret L, Lause P, Grobet L, Attaix D et al. Myostatin gene deletion prevents glucocorticoid-induced muscle atrophy. Endocrinology 2007; 148: 452-460.

31 Urban RJ, Bodenburg YH, Gilkison C, Foxworth J, Coggan AR, Wolfe RR et al. Testosterone administration to elderly men increases skeletal muscle strength and protein synthesis. Am J Physiol 1995; 269(5 Part 1): E820-E826.

32 Wu Y, Zhao W, Zhao J, Pan J, Wu Q, Zhang Y et al. Identification of androgen response elements in the IGF-1 upstream promoter. Endocrinology 2007; 148: 2984-2993.

33 Kovacheva EL, Hikim AP, Shen R, Sinha I, Sinha-Hikim I. Testosterone supplementation reverses sarcopenia in aging through regulation of myostatin, c-Jun $\mathrm{NH2}$ terminal kinase, Notch, and Akt signaling pathways. Endocrinology 2010; 151: 628-638. 